\title{
Virtual reality simulation for the optimization of endovascular procedures: current perspectives
}

\author{
This article was published in the following Dove Press journal: \\ Vascular Health and Risk Management \\ 10 March 2015 \\ Number of times this article has been viewed
}

\section{Nung Rudarakanchana' Isabelle Van Herzeele ${ }^{2}$ Liesbeth Desender ${ }^{2}$ Nicholas JW Cheshire' \\ 'Department of Surgery, Imperial College London, London, UK; ${ }^{2}$ Department of Thoracic and Vascular Surgery, Ghent University Hospital, Ghent, Belgium}

On behalf of EVEREST (European Virtual reality Endovascular RESearch Team)
Correspondence: Nung Rudarakanchana Department of Surgery, St Mary's Hospital, Room I003, QEQM Building, South Wharf Road, London W2 INY, UK Tel +44 2033128120

Email n.rudarakanchana@imperial.ac.uk

\begin{abstract}
Endovascular technologies are rapidly evolving, often requiring coordination and cooperation between clinicians and technicians from diverse specialties. These multidisciplinary interactions lead to challenges that are reflected in the high rate of errors occurring during endovascular procedures. Endovascular virtual reality (VR) simulation has evolved from simple benchtop devices to full physic simulators with advanced haptics and dynamic imaging and physiological controls. The latest developments in this field include the use of fully immersive simulated hybrid angiosuites to train whole endovascular teams in crisis resource management and novel technologies that enable practitioners to build VR simulations based on patient-specific anatomy. As our understanding of the skills, both technical and nontechnical, required for optimal endovascular performance improves, the requisite tools for objective assessment of these skills are being developed and will further enable the use of VR simulation in the training and assessment of endovascular interventionalists and their entire teams. Simulation training that allows deliberate practice without danger to patients may be key to bridging the gap between new endovascular technology and improved patient outcomes.
\end{abstract}

Keywords: virtual reality, simulation, endovascular, aneurysm

\section{Introduction}

The rise in the use of virtual reality (VR) simulation in health care was instigated by the publication of the Institute of Medicine 2000 report "To Err is Human: Building a Safer Health System", ${ }^{1}$ which highlighted the frequency of medical errors and recommended simulation as a tool to improve clinician training and shorten the learning curve without risk of further harm to patients. Simulation training in health care has continued to flourish, in part in response to decreased training opportunities, a change in societal attitude to clinical training, and the need to rapidly attain, refresh, and maintain skill sets.

In most vascular surgery centers, endovascular treatments have now superseded open surgical repair in the management of a large number of pathologies. However, endovascular technologies are relatively novel and rapidly evolving, and often require the coordination of a number of clinicians and technicians from diverse specialties. These challenges are reflected in the higher rate of errors during endovascular phases of hybrid open surgical and endovascular procedures. ${ }^{2}$ This paper reviews the evidence for VR simulation in the optimization of endovascular procedures, with particular reference to the acquisition of both technical and human factor skills, and explores the latest frontiers in this field, discussing the use of immersive VR simulation in training of whole endovascular teams and the novel technologies that now enable 
patient-specific VR rehearsal. Simulation training that allows deliberate practice without danger to patients may be key to bridging the gap between new endovascular technology and improved patient outcomes.

In this paper, we review the current state of endovascular VR simulation, with reference to both technical and nontechnical skills, and describe some of the latest developments in the field, including fully immersive simulation for team training and advanced software that enables patient-specific rehearsal (PsR).

\section{VR simulation}

VR simulation refers to the process of imitating a course of events using computer-generated representation that allows sensory interaction, and may range from patient encounters and plastic modules to VR simulators. The goal of simulation training is to shorten and flatten the learning curve of any surgical procedure, allowing proficiency to be achieved before the procedure is performed on a real patient. Not all VR simulations are fully immersive, hightech interactive environments; indeed, low-fidelity simulations may be useful in the training of novices in basic task acquisition and sequencing, who might otherwise be overwhelmed by advanced technology features and multitask requirements of high-fidelity simulators. Similarly, fully immersive simulators may be of little benefit to novices but provide high-level learning opportunities for experienced practitioners, including training in nontechnical or human factor skills.

In order to achieve satisfactory training in procedural performance, simulation must still be integrated within a full curriculum, with cognitive training completed prior to initiation of practical skills simulation. The validity of simulations may be assessed against a range of parameters (Table 1). ${ }^{3}$ Ideally, simulations should be developed after careful task analysis, involving a multidisciplinary team of experts, with identification of tasks during which most errors

Table I Simulation validity

\begin{tabular}{ll}
\hline Face validity & $\begin{array}{l}\text { Extent to which simulation resembles real life } \\
\text { Content validity }\end{array}$ \\
Extent to which domain being simulated is \\
simulated \\
Extent to which performance on simulator \\
discriminates between novices and experts \\
Concurrent validity & $\begin{array}{l}\text { Extent to which performance on simulator } \\
\text { correlates with gold standard }\end{array}$ \\
Predictive validity & $\begin{array}{l}\text { Ability of performance on simulator to } \\
\text { predict future performance }\end{array}$ \\
Feasibility & Measure of whether simulation is capable of \\
& being carried out
\end{tabular}

occur, and weighting of the errors. The results of such task analysis may then be used to define the assessment parameters or performance metrics, with continued enhancement and refinement following testing. Performance of both technical and nontechnical skills may be objectively assessed. VR simulators may provide automated results of the former, for example, time-action analysis, error analysis, and motion analysis, and both global and specific procedure checklists may be used in conjunction with video recording to further assess technical performance. Nontechnical rating scales, such as the Oxford Non-Technical Skills Scale, ${ }^{4}$ the Mayo High Performance Teamwork Scale, ${ }^{5}$ and the Observational Teamwork Assessment for Surgery (OTAS), ${ }^{6}$ may be used to evaluate human factor skills and whole team performance.

\section{Endovascular VR simulation}

VR simulation training has been shown to improve technical proficiency and shorten learning curves in other fields requiring fine dexterity and hand-eye coordination, such as laparoscopic surgery and endoscopy. ${ }^{7,8}$ In the endovascular arena, VR simulation software is available for cerebral, carotid, coronary, renal, aortic, iliofemoral, and infrageniculate procedures. Simulators usually consist of one or two haptic devices ("legs") connected to one to two LED monitors, displaying tools and a simulated fluoroscopy screen, and a laptop computer, from which a facilitator may select tools as instructed and manipulate physiological parameters, according to the scenario. Wires, catheters, simulated balloons, stent, and stent grafts may be inserted and deployed as in real-life endovascular procedures, and both static and dynamic fluoroscopic imaging may be undertaken, with participants given foot pedals, C-arm, and zoom toggles to control simulated radiological exposure and table movement. Currently, a number of systems are available on the commercial market, including the Vascular Intervention Simulation Trainer (VIST; Mentice, Gothenbrug, Sweden), Angio Mentor (Simbionix, Cleveland, OH, USA), SimSuite (Medical Simulation Corporation, Denver, CO, USA), and CathLabVR (CAE Healthcare, Saint-Laurent, QC, Canada).

Endovascular simulators have been criticized in the past for poor haptic feedback and tactile inaccuracies of guidewire manipulation. However, technology is fast evolving and modern VR simulators benefit from improved haptics and sophisticated software that allows dynamic physiological patient representation according to performance during a task, for example, in a ruptured abdominal aortic aneurysm (rAAA) module, increased blood pressure and decreased 
heart rate upon inflation of an intraaortic balloon supported by a long sheath.

Recently, a full physic, immersive hybrid angiosuite simulator has become available on the commercial market. Although costly and not portable, the Orcamp (Ozone, Gothenburg, Sweden) simulator enables whole team training, including C-arm manipulation and fluoroscopy without exposure to ionizing radiation. When integrated with traditional tabletop VR simulators, the integrated environment allows training of both technical and nontechnical endovascular skills, with expectation of better transferability to real-world procedures.

Endovascular VR simulation training has been shown to improve residents' procedural skills in peripheral angioplasty and stenting ${ }^{9}$ and experienced interventionalists' performances in carotid artery stenting (CAS). ${ }^{10}$ Two studies have reported transferability of skills, with improvement in real-world performance of endovascular procedures for lower extremity occlusive disease following VR training. ${ }^{11,12}$

Evidence suggests that both novices, such as medical students, and those experienced in endovascular procedures may benefit from VR simulation training. ${ }^{13}$ Interestingly, novices were able to quickly improve performance on an endovascular VR simulation (in terms of reduced total procedure time), but further simulation training was insufficient to overcome an evident plateau and performance remained inferior to that of experts in the field. Experienced endovascular interventionalists may use VR simulation to learn new techniques or practice using novel endovascular tools. In a study of experienced endovascular specialists, performance on a VR simulation of CAS was evaluated before and after a CAS training course.${ }^{14}$ Following the course, the subjects performed the CAS procedure faster, with less radiation and fewer errors (movements of guiding catheter/sheath without leading wire, number of spasms of internal carotid artery).

\section{Technical endovascular skills}

The lack of agreed definitions of technical endovascular skills and sufficient tools for objective measurement of such skills remains a significant obstacle in the validation and wider adoption of endovascular VR simulators. Surrogate measures of technical skill, such as time to complete tasks, contrast use, fluoroscopy exposure time, and number of errors (eg, advancement of catheters without guidewires, number of contacts of stiff wire with vessel wall), may not adequately reflect the quality of endovascular skill, and certainly, there is little evidence that improved performance in these metrics correlates with procedural experience or results in superior procedural outcomes. In an early study by our group using the VIST simulator, interventionalists experienced in CAS were arbitrarily divided into four groups based on their clinical CAS experience. ${ }^{15}$ The more experienced specialists performed the simulated intervention faster and used less radiation. However, the levels of CAS experience could not be differentiated using the automated error recordings (clinical tools used, residual stenosis) from the VR simulator. A generic rating scale of basic endovascular skill was therefore developed, focusing mainly on hand movements and using a Likert scale of 1 (very poor performance) to 5 (excellent performance) (Figure 1). Videotaping of the fluoroscopy screen and clinicians' hands allowed post hoc video-based assessment by independent experienced interventionalists; performance on this rating scale was able to differentiate between clinicians of varying CAS experience. Procedure-specific rating scales for various endovascular procedures have also been developed, enabling evaluation of procedure-specific technical endovascular skills using post hoc video-based analysis of fluoroscopy screen images.

Evidence from many VR simulator-based studies indicates that no single simulator-derived metric can confirm that an expert level of proficiency has been acquired. Consequently, assessments of performances on a VR simulator should not solely be based on simulator-derived metrics but instead be combined with additional objective ratings, such as live or video-based evaluations.

More recent work toward objective measurement of technical endovascular skill has involved the development of semi-automated catheter-tracking software with motion analysis of fluoroscopic videos and calculation of $2 \mathrm{D}$ catheter tip path length ${ }^{16}$ and a force/torque sensor allowing measurement of tool forces applied by endovascular operators. ${ }^{17}$ Both platforms have potential to further refine objective assessment of technical endovascular skill performance on VR simulators.

\section{Nontechnical endovascular skills}

It has long been recognized that differentiation between novice and expert performance of surgical procedures is based not solely on degrees of technical skill but also, and perhaps more importantly, on proficiency in nontechnical or human factor skills (Figure 2). Thus, although VR simulation training in the context of health care has traditionally focused on the attainment of technical skills, more contemporary advanced practice is turning toward exploiting the potential of 


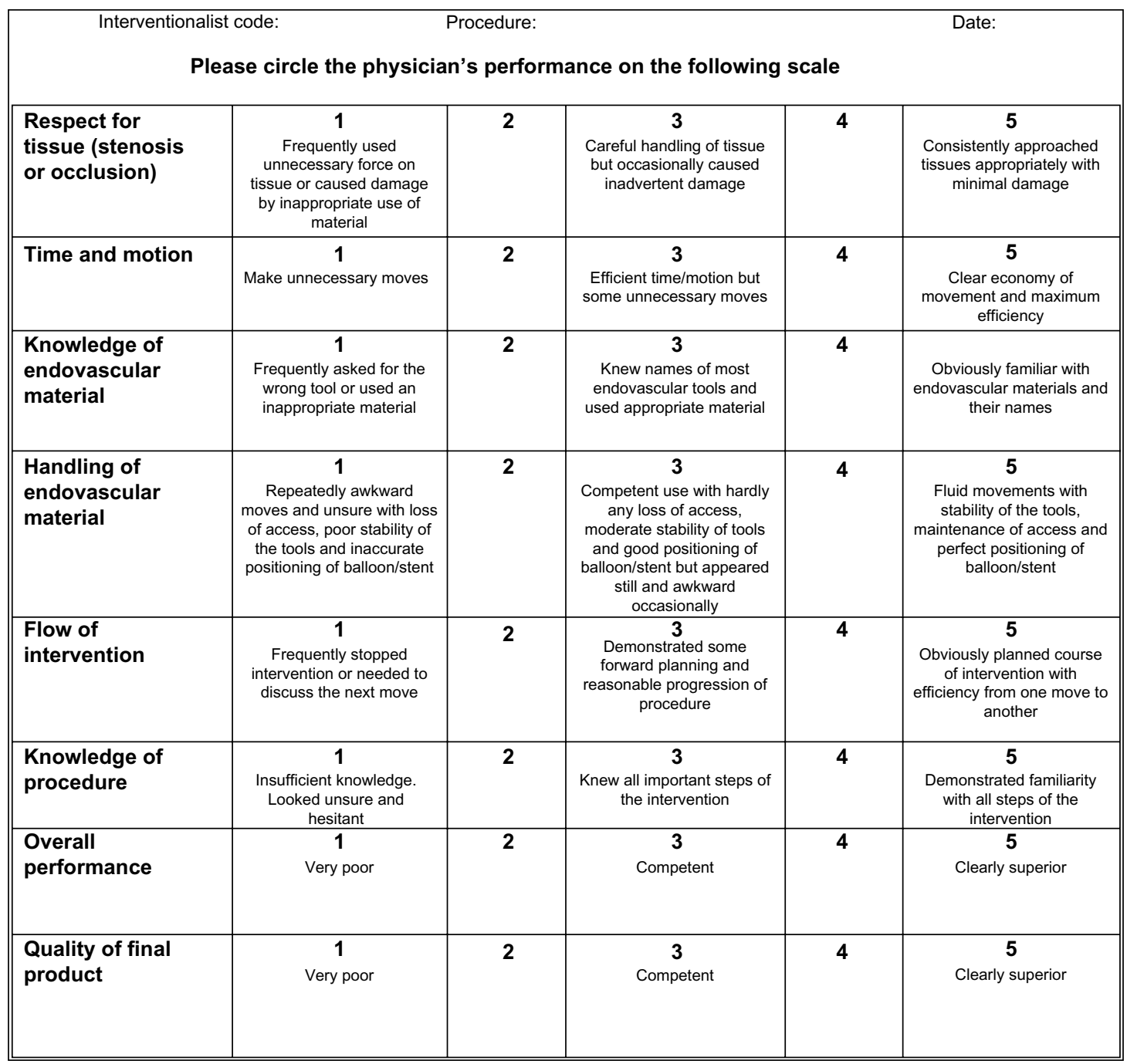

Figure I Modified global rating scale of generic endovascular skills.

fully immersive VR simulators in the training of nontechnical skills and whole teams. ${ }^{18}$ Although costly, such high-fidelity immersive environments may have advantages over other types of simulation, enabling state-dependent learning with a higher rate of real-world skill transfer.

\section{System errors and crisis resource management}

Our understanding of the causes of errors and strategies to mitigate such errors comes from the experience of high-reliability organizations, for example, armed forces aircraft, nuclear power, and air traffic control. Experts in the study of error advocate a systems, rather than a person, approach to understanding and correcting root causes. ${ }^{19}$ Error events occur when active failures (slips, lapses, mistakes, procedural violations) and latent conditions (system faults), visualized as moving points of weakness in a wall of defense, become aligned (the so-called Swiss Cheese effect). Countermeasures are based on system defenses, including barriers and safeguards for hazardous technologies, for example, engineered controls (alarms, physical barriers), people (surgeons, anesthetists, pilots), procedures, and administrative controls.

High-reliability organizations recognize human variability and work to harness this in combination with intelligent wariness in order to avert errors, creating resilient systems. Adaptability is acknowledged as key, with routine modes of working, with conventional hierarchy, combined with a shift of control to experts during crisis mode. There is an open acknowledgment of the possibility of failure, with training focusing on error recognition and prevention, learning through the generalization of failures, and resultant instigation of system reforms. 


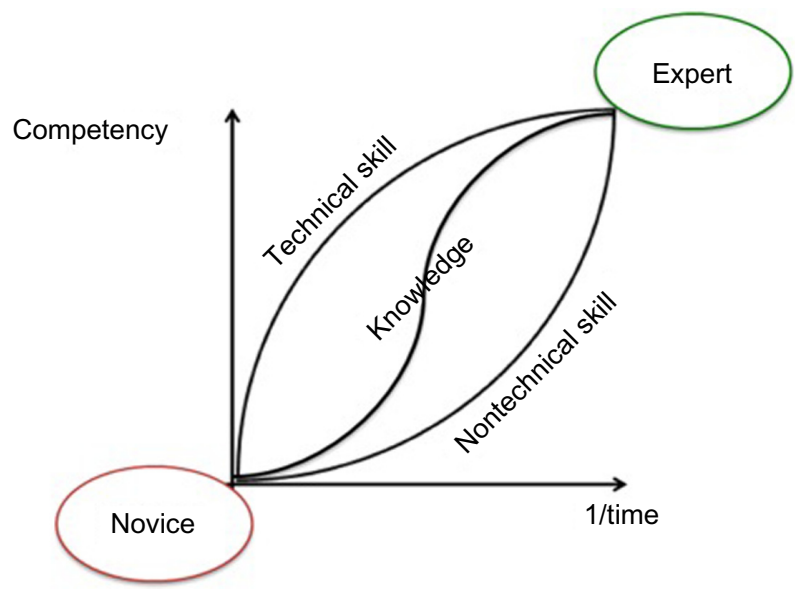

Figure 2 The contributions of knowledge and technical and nontechnical skills in the development from novice to expert performance.

Crisis resource management (CRM) forms part of proactive risk minimization strategy and is a comprehensive approach, targeting people, teams, tasks, workplaces, and whole institutions. ${ }^{20}$ The principles of CRM (Table 2 ) are used to train teams in the functions of leaders and followers, with improved communication, support, and global assessment.

\section{Nontechnical skills}

Generic nontechnical skills comprise both interpersonal and cognitive skills. These domains may be further subdivided as follows.

\section{Interpersonal skills}

i. Communication: share mental model, check hearing and understanding, repeat and acknowledge, active listening.

ii. Team working: cooperation, compensate for any weakness in leadership.

iii. Leadership: direct team member performance toward achieving common goal, construct open environment to allow all team members a voice, information exchange,

Table 2 The principles of crisis resource management

I. Leadership: establish leader, support, and function

2. Emphasis on communication as key skill

3. Planning and anticipation

4. Prioritization

5. Continuous reassessment

6. Intelligent use of all team members and resources available

7. Flexibility and adaptation of ideas and goals within team

8. Optimizing team performance through consideration of personality traits

9. Effective coping with disruptions and distractions "what is right" not "who is right", adapt leadership style.

\section{Cognitive skills}

i. Situational awareness: assess and anticipate, avoid fixation error inherent in concentration, frequent scanning of environment.

ii. Planning: focus on planning as a single task, consider worst-case scenario, plan alternatives and margins of time, resources and staff, consider long-term outcomes.

iii. Decision making: identify options and then act and evaluate, challenge hypotheses, correct mistakes immediately, early call for help, feedback.

iv. Task management.

Patient safety depends on the skills, vigilance, and judgment of expert clinicians. While the majority of team members in other high-stakes organizations are known to each other, the working patterns in modern medical practice mean that health care teams often now comprise unrehearsed groups who are unknown to each other, not familiar with each other's roles, special skills, or goals. These barriers to teamwork may be particularly acute in the emergency surgical situation, for example, in the management of patients with an rAAA. Effective teamwork in these situations therefore requires rapid organization and a unified framework of behaviors, key to team forming. CRM can be used to teach such ad hoc teams, which may include practitioners from several disciplines, to function effectively.

CRM recognizes the barriers to problem solving during crisis and in complex environments, including uncertainty and risk, a lack of transparency, information lack or overload, time pressure, goal plurality, and multiplayer situations, often with strangers.

Simulated environments are ideal for teaching CRM; they may be manipulated to introduce a sense of urgency to emotionally charge and change impact of rehearsed scenarios. Group dynamics, taking into account the individual traits of team members, may be analyzed and reflected upon in detail and adjusted to improve performance. Effective immersive VR simulation environments allow participants to suspend disbelief and buy into the simulation scenario; this is thought to result in stronger imprinting with consequent durable impact on future behavior. CRM is most commonly taught in conjunction with video recording of simulation performances, with structured debriefing, be that on a one-to-one basis or with the whole team, enabling the application of safety concepts, principles, and terminology. Participants 
are encouraged to comment on their own performance, with teachers and facilitators providing supportive, and nonjudgmental, confidential feedback. Interestingly, CRM training has also been shown to improve technical performance in theater and reduce clinical error rates. ${ }^{21,22}$

\section{Fully immersive simulation for training in emergency endovascular procedures}

Current technology allows fully immersive high-fidelity VR simulation of crisis endovascular scenarios to be created and carried out by multidisciplinary teams, enabling whole team training and assessment. Operations with high mortality rates and low error tolerance are also often those with the lowest volumes. This relative lack of real-world procedure experience makes it challenging to maintain the highest levels of performance and optimize patient outcomes, particularly in cases where team members do not work together regularly. Although the proportion of rAAA treated by endovascular means is increasing and mortality rates following endovascular repair of rAAA (rEVAR) have improved, ${ }^{23}$ there is a decline in the availability of appropriately trained teams with consequent inability to perform rEVAR in suitable patients. ${ }^{24}$ Simulation training provides the potential to increase the number of trained teams able to offer endovascular repair to patients with rAAA.

In a recent study, we demonstrated the feasibility of integration of two tabletop VR simulators (VIST-C, Mentice) in Orcamp, creation of a simulated rEVAR scenario with time-dependent triggers, and team performance of this crisis scenario in the fully immersive simulated angiosuite environment (Figure 3). Analysis of objective technical skills assessment parameters (time to: proximal aortic occlusion, deployment of main body of aortic stent graft, cannulation

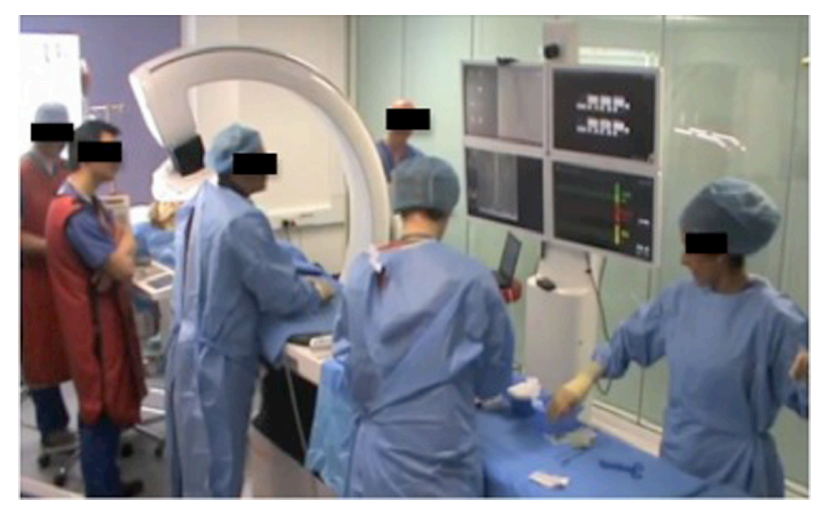

Figure 3 Team training in a fully immersive hybrid angiosuite simulator. of contralateral limb gate, and completion of procedure; total fluoroscopy time) suggested good construct validity for this model, with experienced interventionalists being significantly faster than trainees in achieving critical procedural steps in this crisis scenario. Moreover, the simulation appeared to have good face validity: participants reported that the VR simulators realistically simulated an actual rEVAR procedure. Post-procedural questionnaire responses also suggested that immersive VR simulation of an endovascular crisis scenario had potential utility in the improvement of technical and human factor skills, particularly teamwork, and enhancing patient safety. Trainees valued the simulation higher than experienced endovascular specialists in terms of opportunities for technical skill acquisition, while experienced team leaders saw more benefit for the simulation to improve communication skills. These findings reflect how skills may be attained for complex procedures: less experienced operators initially focus on gaining technical skills, while more experienced operators realize that human factor skills are paramount to enhance overall team performances. ${ }^{25}$ Indeed, most intraoperative errors during endovascular procedures arise from failures in situation awareness, teamwork, and communication skills. ${ }^{26,27}$

There remains a lack of objective assessment tools of nontechnical endovascular skill; such instruments are crucial if we are to provide effective training in this field. Our group is currently working on the development of a teamwork assessment tool, specifically designed for endovascular teams, with modification of the well-validated OTAS to produce Endo-OTAS.

\section{Patient-specific rehearsal}

Representing the next step in the evolution of endovascular simulation, technology is now available to allow VR simulation using patient-specific data. The principles and utility of the so-called procedure rehearsal, or mission rehearsal, are already well recognized in the fields of sports and music and other high-stakes industries. ${ }^{28}$ Briefly, computed tomography or magnetic resonance angiographic images are used to generate $3 \mathrm{D}$ reconstructions using proprietary software. Simulation of vessel wall calcification is automated, and manual augmentation may be used to adjust modeling of vascular branches (eg, visceral, iliac branches). Bony landmarks and vessel centerlines are assigned and used to align the images within the simulation. These images are then uploaded into software on tabletop VR simulators, and interventionalists are able to rehearse the procedure, optimizing treatment through the testing of different approaches and devices, on 
their own or with the entire team. PsR not only facilitates procedure planning (cognitive rehearsal) and technical practice (psychomotor rehearsal) but also enables team rehearsal. The implications for potential improvement in patient safety and costs are self-evident.

The first endovascular PsR case was presented in 2005 and involved VR simulation of CAS using the Simbionix PROcedure rehearsal studio software (Simbionix). Since then, studies have shown the feasibility of this approach, with results suggesting good face validity and the potential to influence choice of endovascular tools, and improve nontechnical skills and patient safety. ${ }^{29-32}$ More recently, software has become available that allows PsR of endovascular abdominal aortic aneurysm repair (EVAR), enabling interventionalists to test the behavior of chosen stent grafts in patient-specific anatomy and identify potential hazards (eg, errors in stent graft sizing, endoleaks) before performing implantation in the real patient (Figure 4). A pilot study has suggested good face validity and the ability to influence fluoroscopy preferences and treatment plans. ${ }^{33}$ Results of a randomized controlled trial to evaluate how EVAR PsR influences technical and team performance are expected shortly. Software to allow PsR of thoracic endovascular aortic repair is now available, although not yet validated in any published study.

Current commercially available endovascular PsR software continues to be refined. Limitations of PsR simulations include the time, expertise, and costs required to generate the simulation reconstructions, reliance on the quality of the axial imaging (CTA or MRA), and inadequate modeling of vessel biomechanical properties, for example, straightening effect of stiff wires, reconfiguration following ballooning, and stent or stent graft deployment. Ongoing collaboration
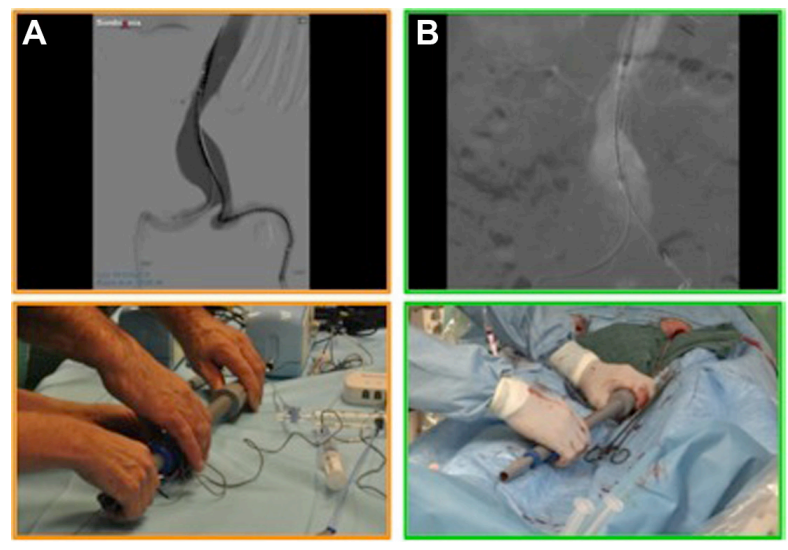

Figure 4 Patient-specific rehearsal of EVAR.

Notes: (A) Simulation. (B) Real-life case.

Abbreviation: EVAR, endovascular abdominal aortic aneurysm repair. between clinicians and industry is making good progress in addressing these concerns.

\section{Conclusion}

Although a still recent technology, endovascular VR simulation has developed a pace over the past decade, with increasingly sophisticated devices and environments now available that provide opportunity to improve not only technical but also human factor skills. There is still work to be done in terms of fully validating the simulations used, but undeniably advanced endovascular VR simulations, such as fully immersive angiosuite scenarios and PsR, have great potential in optimizing endovascular performance and further improving patient outcomes.

\section{Disclosure}

The authors report no conflicts of interest in this work.

\section{References}

1. Kohn LT, Corrigan JM, Donaldson MS, editors. To Err is Human: Building a Safer Healthcare System. Washington, DC: National Academy Press; 1999.

2. Patel SR, Gohel MS, Hamady M, et al. Reducing errors in combined open/endovascular arterial procedures: influence of a structured mental rehearsal before the endovascular phase. J Endovasc Ther. 2012;19(3): 383-389.

3. Aggarwal R, Grantcharov T, Moorthy K, et al. An evaluation of the feasibility, validity, and reliability of laparoscopic skills assessment in the operating room. Ann Surg. 2007;245(6):992-999.

4. Mishra A, Catchpole K, McCulloch P. The Oxford NOTECHS system: reliability and validity of a tool for measuring teamwork behaviour in the operating theatre. Qual Saf Health Care. 2009;18(2):104-108.

5. Malec JF, Torsher LC, Dunn WF, et al. The mayo high performance teamwork scale: reliability and validity for evaluating key crew resource management skills. Simul Healthc. 2007;2(1):4-10.

6. Hull L, Arora S, Kassab E, Kneebone R, Sevdalis N. Observational teamwork assessment for surgery: content validation and tool refinement. J Am Coll Surg. 2011;212(2):234-243. e1-e5

7. Seymour NE, Gallagher AG, Roman SA, et al. Virtual reality training improves operating room performance: results of a randomized, doubleblinded study. Ann Surg. 2002;236(4):458-463.

8. Walsh CM, Sherlock ME, Ling SC, Carnahan H. Virtual reality simulation training for health professions trainees in gastrointestinal endoscopy. Cochrane Database Syst Rev. 2012;6:CD008237.

9. Dawson DL, Meyer J, Lee ES, Pevec WC. Training with simulation improves residents' endovascular procedure skills. J Vasc Surg. 2007;45(1):149-154.

10. Van Herzeele I, Aggarwal R, Neequaye S, et al. Experienced endovascular interventionalists objectively improve their skills by attending carotid artery stent training courses. Eur J Vasc Endovasc Surg. 2008;35(5): 541-550.

11. Chaer RA, Derubertis BG, Lin SC, et al. Simulation improves resident performance in catheter-based intervention: results of a randomized, controlled study. Ann Surg. 2006;244(3):343-352.

12. Hseino H, Nugent E, Lee MJ, et al. Skills transfer after proficiencybased simulation training in superficial femoral artery angioplasty. Simul Healthc. 2012;7(5):274-281.

13. Van Herzeele I, O'Donoghue KG, Aggarwal R, Vermassen F, Darzi A, Cheshire NJ. Visuospatial and psychomotor aptitude predicts endovascular performance of inexperienced individuals on a virtual reality simulator. J Vasc Surg. 2010;51(4):1035-1042. 
14. Van Herzeele I, Aggarwal R, Neequaye S, et al. Experienced endovascular interventionalists objectively improve their skills by attending carotid artery stent training courses. Eur J Vasc Endovasc Surg. 2008;35(5): 541-550.

15. Van Herzeele I, Aggarwal R, Choong A, Brightwell R, Vermassen FE, Cheshire NJ. Virtual reality simulation objectively differentiates level of carotid stent experience in experienced interventionalists. $J$ Vasc Surg. 2007;46(5):855-863.

16. Rolls AE, Riga CV, Bicknell CD, et al. A pilot study of video-motion analysis in endovascular surgery: development of real-time discriminatory skill metrics. Eur J Vasc Endovasc Surg. 2013;45(5):509-515.

17. Rafii-Taril H, Payne CJ, Riga C, Bicknell C, Lee SL, Yang GZ. Assessment of navigation cues with proximal force sensing during endovascular catheterization. Med Image Comput Comput Assist Interv. 2012;15(pt 2):560-567.

18. Undre S, Koutantji M, Sevdalis N, et al. Multidisciplinary crisis simulations: the way forward for training surgical teams. World J Surg. 2007;31(9):1843-1853.

19. Reason J. Human error: models and management. BMJ. 2000;320(7237): 768-770.

20. Murray WB, Foster PA. Crisis resource management among strangers: principles of organizing a multidisciplinary group for crisis resource management. J Clin Anesth. 2000;12(8):633-638.

21. McCulloch P, Mishra A, Handa A, Dale T, Hirst G, Catchpole K. The effects of aviation-style non-technical skills training on technical performance and outcome in the operating theatre. Qual Saf Health Care. 2009;18(2):109-115.

22. Morey JC, Simon R, Jay GD, et al. Error reduction and performance improvement in the emergency department through formal teamwork training: evaluation results of the MedTeams project. Health Serv Res. 2002;37(6):1553-1581.

23. Mayer D, Aeschbacher S, Pfammatter T, et al. Complete replacement of open repair for ruptured abdominal aortic aneurysms by endovascular aneurysm repair: a two-center 14-year experience. Ann Surg. 2012;256(5):688-695
24. Franks S, Lloyd G, Fishwick G, Bown M, Sayers R. Endovascular treatment of ruptured and symptomatic abdominal aortic aneurysms. Eur J Vasc Endovasc Surg. 2006;31(4):345-350.

25. Vincent C, Moorthy K, Sarker SK, Chang A, Darzi AW. Systems approaches to surgical quality and safety: from concept to measurement. Ann Surg. 2004;239(4):475-482.

26. Morbi AH, Hamady MS, Riga CV, et al. Reducing error and improving efficiency during vascular interventional radiology: implementation of a preprocedural team rehearsal. Radiology. 2012;264(2):473-483.

27. Albayati MA, Gohel MS, Patel SR, Riga CV, Cheshire NJ, Bicknell CD. Identification of patient safety improvement targets in successful vascular and endovascular procedures: analysis of 251 hours of complex arterial surgery. Eur J Vasc Endovasc Surg. 2011;41(6):795-802.

28. Krebs WK, McCarley JS, Bryant EV. Effects of mission rehearsal simulation on air-to-ground target acquisition. Hum Factors. 1999;41(4): $553 \mathrm{e} 8$.

29. Cates CU, Patel AD, Nicholson WJ. Use of virtual reality simulation for mission rehearsal for carotid stenting. J Am Med Assoc. 2007;297(3): 265 e6.

30. Hislop SJ, Hedrick JH, Singh MJ, et al. Simulation case rehearsals for carotid artery stenting. Eur J Vasc Endovasc Surg. 2009;38(6):750e4.

31. Willaert W, Aggarwal R, Harvey K, et al. Efficient implementation of patient-specific simulated rehearsal for the carotid artery stenting procedure: part-task rehearsal. Eur J Vasc Endovasc Surg. 2011;42(2): $158 \mathrm{e} 66$.

32. Willaert W, Aggarwal R, Bicknell C, et al. Patient-specific simulation in carotid artery stenting. JVasc Surg. 2010;52(6):1700e5.

33. Desender L, Rancic Z, Aggarwal R, et al. Patient-specific rehearsal prior to EVAR: a pilot study. Eur J Vasc Endovasc Surg. 2013;45(6): 639-647.
Vascular Health and Risk Management

\section{Publish your work in this journal}

Vascular Health and Risk Management is an international, peerreviewed journal of therapeutics and risk management, focusing on concise rapid reporting of clinical studies on the processes involved in the maintenance of vascular health; the monitoring, prevention and treatment of vascular disease and its sequelae; and the involvement of

\section{Dovepress}

metabolic disorders, particularly diabetes. This journal is indexed on PubMed Central and MedLine. The manuscript management system is completely online and includes a very quick and fair peer-review system, which is all easy to use. Visit http://www.dovepress.com/ testimonials.php to read real quotes from published authors. 